\title{
HONGOS LIQUENIZADOS HALLADOS SOBRE RESTOS ARQUEOLÓGICOS. Nuevos Registros para la provincia de Santa Cruz y un nuevo REgISTRO PARA ARgENTINA
}

\author{
RENATO GARCÍA ${ }^{1,4}$, LUCIANA LABORDA ${ }^{1,2}$, VILMA ROSATO ${ }^{1,5}$ y LUCIA MAGNIN LI, $^{2,5}$
}

\begin{abstract}
Summary: Lichenized fungi found on archaeological remains. New records for the province of Santa Cruz and a new record for Argentina. As part of an anthropological study, the lichenized fungi on the surface of archaeological remains from La Primavera locality in Santa Cruz province, Argentina, were identified. To achieve this, external morphological characters were observed and handmade cuts of apothecia were observed under light microscope; also the spot tests with K (potassium hydroxide), C (sodium hypochlorite) and reaction under UV light were performed. As a result, 16 species of which 2 previously cited for the province, 13 are new records for the province and Carbonea vorticosa is a new record for the country. Identifying species that grow on this type of anthropogenic substrate is relevant both for future floristic, ecological and biogeographical studies as well as for archaeological studies.
\end{abstract}

Key words: Carbonea vorticosa, archeological remains, Santa Cruz, lichenized fungi.

\begin{abstract}
Resumen: Como parte de un estudio de carácter antropológico se identificaron los hongos liquenizados sobre restos arqueológicos de superficie provenientes de la localidad La Primavera de la provincia de Santa Cruz, Argentina. Para esto se observaron caracteres morfológicos externos y cortes a mano alzada de apotecios que fueron observados bajo microscopio óptico; además se realizaron pruebas con reactivos $\mathrm{K}$ (hidróxido de potasio), C (hipoclorito de sodio) y reacción bajo luz ultravioleta. Como resultado, se encontraron 16 especies de las cuales 2 se encontraban previamente citadas para la provincia, 13 son nuevas citas para la provincia y Carbonea vorticosa es un nuevo registro para el país. La identificación de las especies que crecen sobre este tipo de sustratos es de suma importancia para futuros estudios ecológicos, florísticos, biogeográficos y arqueológicos.
\end{abstract}

Palabras clave: Carbonea vorticosa, restos arqueológicos, Santa Cruz, hongos liquenizados.

\section{INTRODUCCIÓN}

Los hongos liquenizados, conocidos vulgarmente como líquenes son organismos que presentan una gran resistencia a condiciones ambientales extremas, se los puede encontrar en casi cualquier

\footnotetext{
${ }^{1}$ Laboratorio de Entrenamiento Multidisciplinario para la Investigación Tecnológica (1900) La Plata elrenakpo@ yahoo.com.ar

${ }^{2}$ Facultad de Ciencias Naturales y Museo (UNLP) (1900) La Plata.

${ }^{3}$ Centro de Investigaciones Viales (LEMAC) UTN, FRLP (1900) La Plata.

${ }^{4}$ CIC-BA

${ }^{5}$ CONICET
}

ecosistema terrestre, y sobre una gran variedad de sustratos. En zonas áridas donde las plantas vasculares no son tan numerosas, los líquenes pueden llegar a cubrir grandes zonas de los suelos y roquedales, donde la mayoría presentan una morfología crustosa, si bien no es exclusiva de este tipo de ambientes (Rogers, 1977). Los arqueólogos en su interés de obtener información cronológica, y/o de procesos de formación, los han utilizado para datar distintos eventos de interés, como la construcción de monumentos, el uso de canteras de extracción de rocas, la construcción de estructuras de piedra y la realización de arte rupestre (Bettinger \& Oglesby, 1985; Albeck, 1993, 1996; Benedict, 2009; Borrero et al., 2011; Garibotti et al., 2011). 
Otro uso es el análisis de la distribución de los mismos en la superficie de estructuras de piedra para reconocer contextos disturbados de otros no disturbados (Benedict, 2009; Magnin, 2010). Por último, también permiten inferir condiciones generales de estabilidad en la historia postdepositacional de conjuntos arqueológicos de superficie (Borrazo 2011; Borrero et al., 2011; Garibotti et al., 2011; Magnin, 2010).

La localidad La Primavera (situada entre los paralelos $47^{\circ} 48^{\prime}$ y $47^{\circ} 54^{\prime}$ de latitud Sur y los meridianos $68^{\circ} 54^{\prime}$ y $69^{\circ} 2^{\prime}$ de longitud Oeste) fue ocupada por sociedades de cazadores recolectores entre $9518 \pm 64$ y $939 \pm 59$ años AP (Miotti et al., 2007; Magnin, 2010) que dejaron evidencias materiales que se preservaron en superficie. Estos son principalmente artefactos líticos que se encuentran aislados o formando conjuntos de distintas densidades y han servido como sustratos para una amplia variedad de líquenes saxícolas. La misma se localiza dentro de la provincia geológica Macizo del Deseado que se extiende entre el río Deseado y el río Chico de Santa Cruz (Argentina) (Ramos, 1999). Geomorfológicamente es un paisaje mesetiforme, de extensas planicies, las más elevadas están coronadas por mantos basálticos que protegen a las unidades sedimentarias inferiores, observándose en sus márgenes paredones abruptos que suelen rodearse de deslizamientos rocosos (Panza et al. 1994). Fitogeográficamente pertenece al distrito de la Patagonia central de la provincia de Santa Cruz, con una fisonomía de estepa representada por las especies Chuquiraga avellanedae, Nassauvia glomerulosa, Stipa humilis, Jarava neaeiy Pappostipa speciosa (Cabrera, 1971).

Como parte de un proyecto sobre la utilidad de los líquenes en estudios de cronología y tafonomía aplicados a restos arqueológicos (Magnin et al., 2012, 2013), se notó que algunos líquenes hallados sobre ellos no se encontraban citados en los registros nacionales y provinciales. Este trabajo tiene como objetivo citarlos formalmente para contribuir al conocimiento de la biota liquénica de Argentina.

\section{Materiales y Métodos}

Los restos arqueológicos recolectados en la localidad La Primavera son artefactos tallados en piedra que constituyen evidencias materiales de las actividades realizadas por los cazadores recolectores que ocuparon el área en el pasado.

El conjunto analizado está constituido por artefactos líticos entre los cuales se encuentran núcleos, artefactos no formatizados como desechos de talla y lascas, así como por artefactos formatizados tales como raspador, raedera y bola de boleadora (Aschero, com. pers.); un elemento óseo (falange de guanaco) y una muestra de ocre, estos últimos se encontraban en asociación con conjuntos arqueológicos. Se utilizó un microscopio estereoscópico para la observación de la morfología externa y se realizaron cortes a mano alzada de apotecios que fueron observados bajo microscopio óptico de cámara clara. Se realizaron las pruebas puntuales con los reactivos hidróxido de potasio $(\mathrm{K})$ e hipoclorito de sodio (C), además de la reacción bajo luz ultravioleta $(50 \mathrm{~Hz})$. En el caso del género Lecanora se utilizó un microscopio con luz polarizada para observar la ubicación de cristales dentro del apotecio (Śliwa, 2007). Para la determinación se utilizaron las claves de: Malme (1926, 1927), Lamb (1958), Nash et al.(1995), McCune (2000), Ovstedal\& Lewis-Smith (2001), Rambold et al. (2001), Elix (2011a, b), Vondrák et al.(2011) y de la Rosa et al. (2012).

La distribución para Argentina fue tomada de Calvelo \& Liberatore (2002), mientras que la distribución mundial se obtuvo de (http:// www.biologie.uni-hamburg.de/checklists/lichens/ portalpages/portalpage_checklists_switch.htm). En cuanto a las especies del género Lecanora, su distribución mundial se obtuvo de Śliwa (2007) y su distribución en Argentina de de la Rosa et al. (2012).

El material examinado fue depositado en el herbario del Instituto de Botánica "Carlos Spegazzini” (LPS), el coleccionista de la totalidad del material es uno de los autores (L. Magnin). El material lítico fue identificado bajo lupa por personal especializado del LEMIT.

\section{Resultados}

Se encontraron en total 16 especies de líquenes. De estas solo una se encontraba citada para la provincia de Santa Cruz, 14 se encontraban 
registradas previamente en otras provincias de Argentina y una de ellas constituye un nuevo registro para el país. A continuación se presenta un listado ordenado alfabéticamente con las especies identificadas con su correspondiente número de LPS, se agrega una breve descripción morfológica, la distribución conocida y se describe petrológicamente los artefactos líticos sobre los que fueron encontrados.

Amandinea punctata (Hoffm.) Coppins \& Schneid., Lichenologist 25(4): 343. 1993.

Talo inconspicuo, apotecios lecideinos, sésiles de 0,2-0,6 $\mathrm{mm}$ de diámetro, con el disco negro plano y epruinoso, K- C-. El hipotecio es de color marrón. Ascos con 8 esporas, bicelulares elipsoides de color marrón, 8-20 x 5-9 $\mu \mathrm{m}$.

Especie de distribución mundial. Distribución en Argentina: Chubut, Tierra del Fuego, Islas Malvinas y del Atlántico Sur.

Material examinado: ARGENTINA; Santa Cruz; La Primavera; 2010; Magnin L. s.n.; (LPS 48650).

Sustrato: ópalo.

Buellia aethalea (Ach.) Th. Fr., Lich. Scand. (Uppsala) 1: 604. 1874.

Talo crustoso, continuo a rimoso-areolado de color gris blanquecino, epruinoso, protalo conspicuo de color negro K- C-. Apotecios inmersos de 0,1-0,7 $\mathrm{mm}$ de ancho, disco negro, epruinoso, con margen propio reducido. Ascos con 8 esporas, bicelulares elipsoides marrones 11-18 x 6-10 $\mu \mathrm{m}$.

Especie de distribución mundial. Distribución en Argentina: Chubut.

Material examinado: ARGENTINA; Santa Cruz; La Primavera; 2010; Magnin L. s.n.; (LPS 48651).

Sustrato: ópalo.

Buellia subtabacina Malme., Zahlbruckner's Cat. Lich. Univ. 7: 421. 1927. (Fig.1)

Talo crustoso, areolas muy pequeñas, chatas oscuras, K- C-. Apotecios de 0,3-0,5 mm diámetro, sésiles, algo convexos, lecideinos, de color negro. 8 esporas por asco, esporas marrones, elípticas ligeramente curvas, uniseptadas de12-15 x 6-7 $\mu \mathrm{m}$.

Especie sudamericana. Distribución en Argentina: Chubut.

Material examinado: ARGENTINA; Santa Cruz;

La Primavera; 2010; Magnin L. s.n.; (LPS 48652).

Sustrato: toba riolítica y sobre ópalo.
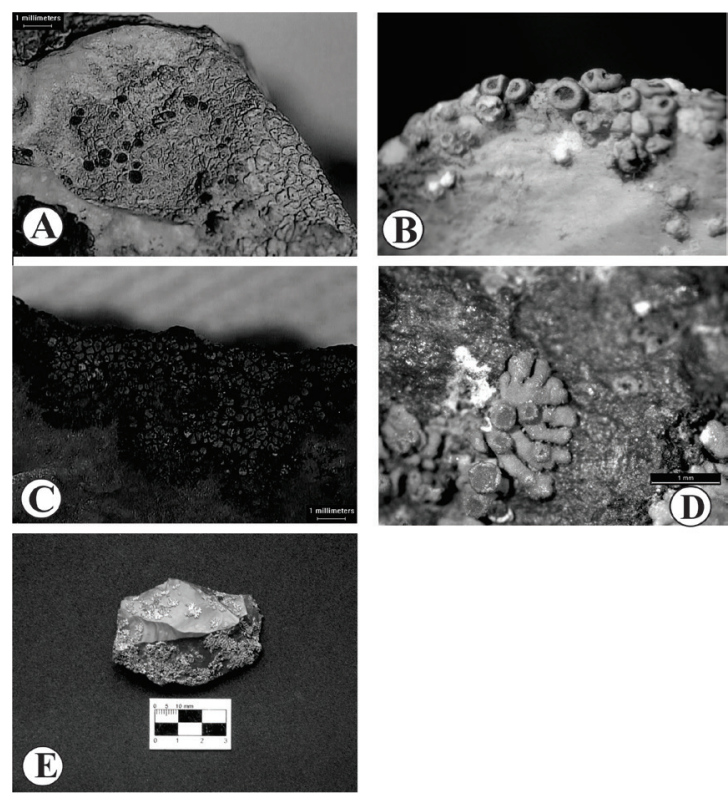

Fig. 1. Líquenes encontrados sobre restos arqueológicos; Buellia subtabacina talo fértil con apotecios de color oscuro (A), apotecios de $L$. wetmorei el talo es muy reducido y endolítico foto obtenida con un aumento de 100X (B); talo de Rhizocarpon disporum creciendo sobre un borde tallado (C); talo fértil, crustoso de bordes lobulados de $X$. elegans (D); talo folioso de Xanthoparmelia. xanthomelanea creciendo sobre material tallado.

Caloplaca cerinella (Nyl.) Flagey, in Battandier \& Trabut, Fl. Algér. crypt. 2(1): 31. 1896.

Talo endolítico, inconspicuo, apotecios lecideinos de color naranja amarillento de 0,2-0,4 $\mathrm{mm}$ de ancho, $\mathrm{K}+$ violeta. Esporas hialinas, no septadas, polariloculares con 12-16 esporas por asco, de 10-13 x 6-7 $\mu \mathrm{m}$.

Especie cosmopolita. Distribución en Argentina: Mendoza.

Material examinado: ARGENTINA; Santa Cruz; La Primavera; 2010; Magnin L. s.n.; (LPS 48654).

Sustrato: cuarcita sedimentaria.

Calopla capuiggari (Müll. Arg.) Zahlbr., Cat. Lich. Univers. 7: 168. 1931.

Talo crustosoareolado, areolas chatas color grisáceo, $\mathrm{K}+$ amarillo tenue C-. Apotecios de 0,7-1 mm de diámetro sésiles, lecanorinos, con disco plano de color naranja, $\mathrm{K}+$ purpura. 8 esporas por asco, hialinas elipsoides no septadas, 
polariloculares de 10-13 x 6-7 $\mu \mathrm{m}$.

Es una especie sudamericana. Distribución en Argentina: Buenos Aires.

Material examinado: ARGENTINA; Santa Cruz;

La Primavera; 2010; Magnin L. s.n.; (LPS 48653).

Sustrato: toba riolítica y sobre ópalo.

Carbonea vorticosa (Flörke) Hertel, Mitt. bot. StSamml., Münch.19: 442. 1983. (Fig. 2)

Talo endolítico, apotecios de hasta $0,6 \mathrm{~mm}$, sésiles con la base constricta, disco negro y márgenes elevados del mismo color, K- C-. Himenio azulado, hipotecio marrón oscuro. Ascos con 8 esporas, hialinas y uniloculares de 10-12 x5-6 $\mu \mathrm{m}$.

Especie de distribución bipolar. No está registrada para Argentina.

Material examinado: ARGENTINA; Santa Cruz; La Primavera; 2010; Magnin L. s.n.; (LPS 48656)

Sustrato: ópalo.

Ingvariella bispora (Bagl.) Guderley \& Lumbsch, in Guderley, Lumbsch \& Feige, Nova Hedwigia 64(1-2): 152. 1997.

Talo crustosoareolado, areolas poligonales planas de color marrón, K- C-, apotecios de 1,5 $\mathrm{mm}$ de diámetro con margen talino y disco negro, esporas muriformes de $28-42 \times 14-23 \mu \mathrm{m}$, color marrón, 1 o 2 por asco.

Especie subcosmopolita. Distribución en Argentina: Tucumán y Buenos Aires.

Material examinado: ARGENTINA; Santa Cruz; La Primavera; 2010; Magnin L. s.n.; (LPS 48657)

Sustrato: ópalo.

Lecanora hagenii (Ach.) Ach., Lich. univ.: 367. 1810

Talo endolítico, apotecios lecanorinos de 0.3-0.9 $\mathrm{mm}$ de diámetro, disco oscuro sin pruina, margen gris pruinoso prominente y discontinuo, $\mathrm{K}-\mathrm{C}$-, anfitecio con cristales visibles bajo luz polarizada. Esporas elipsoides, hialinas, uniloculares, 8 por asco, entre 8-14 x 4-7 $\mu \mathrm{m}$.

Especie cosmopolita. Distribución en Argentina: Buenos Aires y Tierra del Fuego.

Material examinado: ARGENTINA; Santa Cruz; La Primavera; 2010; Magnin L. s.n.; (LPS 48659).

Sustrato: hueso.

Lecanora flowersiana H. Magn., Lamb's Index nom. lich.: 306. 1952.
Talo endolítico, apotecios lecanorinos de 0.6-1.3 $\mathrm{mm}$ diámetro, constricto en la base, agrupados, disco color marrón epruinoso de margen discontinuo epruinoso, K- C-, no se observaron cristales bajo luz polarizada. Con 8 esporas por asco, elipsoides, hialinas de 8-10,5 x 4-6 $\mu \mathrm{m}$.

Especie Americana. Distribución en Argentina: Mendoza.

Material examinado: ARGENTINA; Santa Cruz; La Primavera; 2010; Magnin L. s.n.; (LPS 48660).

Sustrato: ópalo.

Lecanora wetmorei Śliwa, Lichen Flora of the Greater Sonoran Desert Region (Tempe) 2: 283.2004. (Fig. 1)

Talo endolítico, apotecio lecanorino, de 0,4-1,1 $\mathrm{mm}$ de diámetro, sésil, con un margen grisáceo continuo, disco color negro cubierto por una gran cantidad de pruina blanca, K- C-, anfitecio con cristales visibles bajo luz polarizada. Con 8 esporas por asco, elipsoides, hialinas de 9-13 x 5-6,5 $\mu \mathrm{m}$.

Especie encontrada en América e Irán. Distribución en Argentina: Neuquén.

Material examinado: ARGENTINA; Santa Cruz; La Primavera; 2010; Magnin L. s.n.; (LPS 48658).

Sustrato: ópalo.

Rhizocarpon disporum (Nägeli ex Hepp) Müll. Arg., Revue mycol., Toulouse 1: 170. 1879. (Fig. 1)

Talo crustosoareolado, areolas aplanadas o algo convexas de color gris, con un protalo negro, K- CUV-. Apotecios de 0,4-0,9 mm de diámetro convexos sésiles de color negro, sin margen aparente. Ascos uniesporados, esporas muriformes verdes de 48-78 x 18-33 $\mu \mathrm{m}$.

Especie de distribución mundial. Distribución en Argentina: Buenos Aires, Chubut, Mendoza, Río Negro, Santa Cruz y Tierra del Fuego.

Material examinado: ARGENTINA; Santa Cruz; La Primavera; 2010; Magnin L. s.n.; (LPS 48661).

Sustrato: granodiorita, ópalo y toba riolítica ácida.

Umbilicaria decussata (Vill.) Zahlbr., Cat. Lich. Univers. 8: 490. 1932.

Talo folioso umbilicado, hasta $10 \mathrm{~cm}$ de diámetro, color negro, zona del umbón más clara, superficie granulada con arrugas elevadas que conforman una red, sin ricinas, K- C-. Apotecios raros, concoloros con el talo de hasta $3 \mathrm{~mm}$ de diámetro. Esporas simples, 8 por asco. $8-12 \times 5-7 \mu \mathrm{m}$. 
Especie cosmopolita. Distribución en Argentina: Tucumán y Río Negro.

Material examinado: ARGENTINA; Santa Cruz; La Primavera; 2010; Magnin L. s.n.; (LPS 48664).

Sustrato: ópalo.

Umbilicaria polyphylla (L.) Baumg., Fl. Lips.: 571. 1790.

Talo folioso umbilicado hasta $5 \mathrm{~cm}$ de diámetro, con superficie lisa color pardo oscuro, en el sector del umbón presenta una coloración más clara, superficie inferior lisa y sin ricines, K- C-. No se encontraron apotecios.

Especie cosmopolita. Distribución en Argentina: Islas Malvinas e islas del Atlántico Sur.

Material examinado: ARGENTINA; Santa Cruz; La Primavera; 2010; Magnin L. s.n.; (LPS 48663).

Sustrato: toba riolítica.

Xanthoria elegans (Link) Th. Fr., Lich. Arctoi 3: 69. 1860. (Fig. 1)

Talo crustoso, placoide, lobulado en los bordes de color naranja, $\mathrm{K}+$ púrpura. Corteza inferior blanca, apotecios de $3 \mathrm{~mm}$ de diámetro, disco y margen de color coincidente al talo $\mathrm{K}+$ púrpura. 8 esporas por asco, esporas hialinas elipsoides, no septadas, polariloculares, de 11-18 x 5,5-8,5 $\mu \mathrm{m}$.

Especie cosmopolita. Distribución en Argentina: Chubut, Santa Cruz, Tierra del Fuego, Islas Malvinas e islas del Atlántico Sur.

Material examinado: ARGENTINA; Santa Cruz; La Primavera; 2010; Magnin L. s.n.; (LPS 48665).

Sustrato: ocre con hidróxido de hierro.

Xanthoparmelia hypopsila (Müll. Arg.) Hale.

Talo folioso amarillo, adnato al sustrato lóbulos de1-1,5 mm, los sectores viejos se tornan oscuros, médula blanca $\mathrm{K}+$ amarilla tornándose a rojo oscuro, envés negro con ricines las partes más viejas se tornan oscuras. El material estudiado no presentaba apotecios.

Especie cosmopolita. Distribución en Argentina: Buenos Aires, San Luis y Córdoba.

Material examinado: ARGENTINA; Santa Cruz; La Primavera; 2010; Magnin L. s.n.; (LPS 48666).

Sustrato: toba riolítica ácida y roca sedimentaria calcítica.

Xanthoparmelia xanthomelaena (Müll. Arg.) Hale., Phytologia 28: 488. 1974.(Fig. 1)
Talo folioso amarillo, medula blanca $\mathrm{K}+$ naranja, muy adherido al sustrato, lóbulos de $0,5 \mathrm{~mm}$ sin propágulos vegetativos, envés color negro con ricines. Apotecios de 0,5- $1,5 \mathrm{~cm}$ de diámetro, lecanorinos, con el disco negro. 8 esporas por asco, hialinas simples y circulares de $6 \times 10 \mu \mathrm{m}$.

Especie sudamericana, Distribución en Argentina: Chubut.

Material examinado: Argentina; Santa Cruz; La Primavera; 2010; Magnin L. s.n.; (LPS 48667)

Sustrato: ópalo.

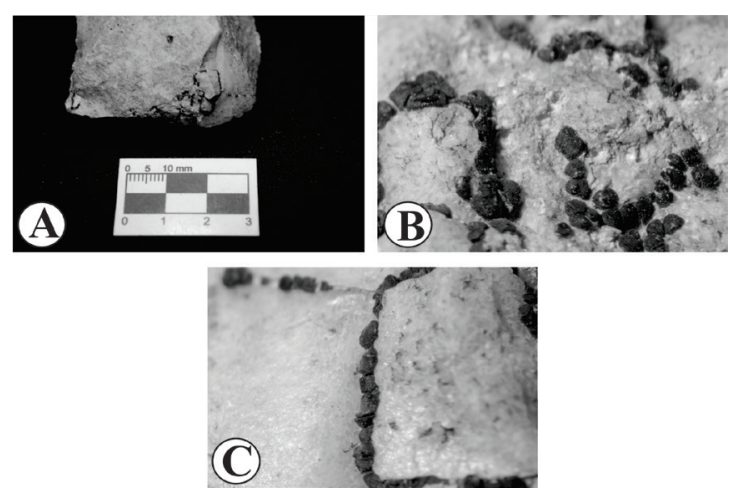

Fig. 2. Morfología externa de Carbonea vorticosa, apotecios sobre el resto arqueológico (A); detalle de los apotecios creciendo entre grietas del material, fotos obtenidas con un aumento de 100X (B y C).

\section{Conclusiones}

Los artefactos arqueológicos han presentado una alta riqueza de líquenes. Sobre ellos se encontraron 16 especies de líquenes de las cuales solo $R$. disporum y $X$. elegans se encontraban previamente citadas para la provincia de Santa cruz (Calvelo\&Liveratore 2002). Las especies $A$. punctata, B. aethalea, B. subtabacina, C. vorticosa, L. hagenii, L. wetmorei, $R$. disporum, U. decusata, $U$. polyphyllay $X$. xanthomelaena son nuevos registros para la provincia de Santa Cruz. Es de destacar que estas especies se encontraban citadas para las provincias aledañas, mientras que para las especies C. cerinella, C. puiggarii, I. bispora, L. flowersiana y $X$. hypopsilaque estaban registradas para el centro y norte de Argentina, ahora su distribución conocida se extiende hasta la provincia de Santa Cruz. 
El nuevo registro para el país, C. vorticosa es descripta como una especie de zonas frías y de distribución bipolar (Ovstedal \& LewisSmith, 2001), en Sudamérica se encontraba citada sólo para Chile (Galloway \& Quilhot, 1998). En Argentina es posible que su distribución sea mayor, pero por su pequeño tamaño es muy probable que pase desapercibida.

La identificación de las especies que crecen sobre este tipo de sustratos es de suma importancia para contribuir con las investigaciones arqueológicas mediante futuros análisis tanto tafonómicos, de liquenometría o como indicadores de condiciones de estabilidad de superficies, para el análisis del estado de conservación de las piezas, como así también para estudios de índole florísticos, ecológicos y biogeográficos.

\section{Agradecimientos}

Se agradece a V. Lynch y a G. Coelho Dos Santos por las fotografías en microscopio y lupa. A Ribot A. por la identificación de los sustratos. Se agradece por el financiamiento parcial del proyecto a CONICET y a la Comisión de investigaciones Científicas de la provincia de Buenos Aires.

\section{Biblografía}

ALBECK, M. E. 1993. Contribución al estudio de los sistemas agrícolas prehispánicos de Casabindo (Puna de Jujuy). Tesis doctoral inédita, Facultad de Ciencias Naturales y Museo, Universidad Nacional de la Plata, La Plata.

ALBECK, M.E. 1996. Utilización de la liquenometría como indicador cronológico en las estructuras agrícolas prehispánicas de Coctaca. Shincal 5: 6779.

BENEDICT, J. B. 2009. A review of lichenometric dating and its applications to archaeology. $\mathrm{Am}$ Antiq. 74: 143-172.

BETTINGER, R. L. \& R. OGLESBY. 1985. Lichen dating of Alpine villages in the White Mountains, California. J. Calif. Gt. Basin Anthropol.7: 202-224.

BORRERO, L. A., K. BORRAZZO, I. GARIBOTTI \& M.C. PALLO. 2011. Concentraciones de pilas de rocas en la cuenca superior del río Santa Cruz (Argentina). Magallania 39:193-206.

CABRERA, A. L. 1971. Fitogeografía de la República Argentina. Bol. Soc. Argent. Bot. 14: 1-42.
CALVELO, S. \& S. LiBERATORE. 2002. Catálogo de los líquenes de la Argentina. Kurtziana 29: 7-170.

DE LA ROSA I. N., M. I. MESSUTI \& L. ŚLIWA. 2012. The Lecanora dispersa group (Lecanoraceae) in Argentina. The Lichenologist 44: 101-114.

ELIX J.A. 2011a. Amandinea, Australian Physciaceae (Lichenized Ascomycota) [online]. Disponible en: http://www.anbg.gov.au/abrs/lichenlist/Amandinea. pdf [Acceso: 20 julio 2013].

ELIX J.A. 2011b. Buellia auct.non De Not., Australian Physciaceae (Lichenized Ascomycota) [on line]. Disponible en:http://www.anbg.gov.au/abrs/ lichenlist/Buellia_SL.pdf [Acceso: 20 julio 2013].

GALLOWAY, D. J. \& W. QUILHOT. 1998. Checklist of Chilean lichen-forming and lichenicolous fungi. Gayana, Bot. 55: 111-185.

GARIBOTTI, I., K. BORRAZZO \& L. A. BORRERO. 2011. Aplicación de técnicas liquenométricas en la arqueología de Patagonia utilizando Rhizocarpon sub género Rhizocarpon. En: Borrero, L.A. \&Borrazzo, K. (eds.) BOSQUES, MONTAÑAS Y CAZADORES Investigaciones Arqueológicas en Patagonia Meridional, pp. 85-104. Editorial Dunken, Capital Federal.

LAMB, I. M. 1958. La vegetación liquénica de los Parques Nacionales Patagónicos. Anales Parques Nac. 7. Buenos Aires.

MAGNIN, L. 2010. Distribuciones arqueológicas en la Meseta central de Santa Cruz. Implicancias para los estudios de uso del espacio y movilidad de sociedades cazadoras recolectoras, Tesis doctoral de Ciencias Naturales, Facultad de Ciencias Naturales y Museo, Universidad Nacional de La Plata, La Plata.

MAGNIN, L., V. ROSATO \& R. GARCÍA. 2012. Información acerca de los líquenes desarrollados sobre artefactos líticos de superficie. Localidad La Primavera, Santa Cruz (Argentina). [on line]. Disponible en: http://www.scha.cl/cnach2012/ info_resumen.php?id=377.

MAGNIN L., R. GARCÍA \& L. LABORDA. 2013. Uso de líquenes para evaluar procesos de formación de sitios de superficie. Un caso en Patagonia extraandina. Libro de Resúmenes. XVIII Congreso Nacional de Arqueología Argentina, p. 200. Universidad Nacional de La Rioja, 22 al 26 de Abril.

MALME, G.O.A. 1926. Lichenes blasteniospori herbarii regnelliani. Arkiv för Botanik 20A : 1-51.

MALME, G.O.A. 1927. Buelliae itineris regnelliani Primi. Arkiv för Botanik 21A : 1-42.

MCCUNE, B. 2011.Caloplaca in the Pacific Norwest. [on line]. Disponible en: http://bmccune.weebly. com/uploads/2/4/5/6/24567979/caloplaca. pdf[Acceso: 20 julio 2013]. 


\section{R. García et al. - Hongos liquenizados hallados sobre restos arqueológicos}

MIOTTI, L., D. HERMO, L. MAGNIN, N. CARDEN, L. MARCHIONNI, A. ALCARAZ, B. MOSQUERA, E. TERRANOVA \& M. SALEMME. 2007. Resolución e integridad arqueológica de la cueva Maripe (Santa Cruz, Argentina). En: MORELLO F., M. MARTINIC, A. PRIETO \& G. BAHAMONDE (eds.), Arqueología de fuego-Patagonia. Levantando piedras desenterrando huesos... y develando arcanos, pp. 555-569. Punta Arenas, Ediciones CEQUA.

NASH, T., C. GRIES \& J. ELIX. 1995. A revision of the Lichen Genus Xanthoparmelia in South America. Biblioth. Lichenol. 56.

OVSTEDAL D.O. \& R.I. LEWIS-SMITH. 2001. Lichens of Antarctica and South Georgia, A guide for their identification and ecology. Cambridge University Press, Cambridge.

PANZA, J., M. ZUBIA, A. GENINI \& M. GODEAS. 1994. Hoja Geológica Tres Cerros 4969-II, Provincia de Santa Cruz. Instituto de Geología y Recursos Minerales, Boletín $n^{\circ}$. 258, SEGEMAR, Buenos Aires.
RAMBOLD, G.,J.A. ELIX, T.H. NASH III, \& L. ZEDDA (eds.), 2001 onwards. LIAS light, a database for rapid identification of lichens [on line]. Disponible en: http://www.liaslight.lias.net/. [Acceso: $18 \mathrm{de}$ febrero de 2014].

RAMOS, V. A. 1999. Rasgos estructurales del territorio argentino. Geolog. Argent. Anales 29: 715-784.

ROGERS, R.W. 1977. Lichens of hot arid and semi-arid lands. In: SEAWARD M. R.D (ed.), Lichen Ecology, pp. 211-251. Academic Press, London.

ŚLIWA, L. 2007. A revision of the Lecanora dispersa complex in North America. Polish Bot. J. 52: 1-70.

VONDRÁK, J., KHODOSOVTSEV, A., ŠOUN, J., \& VONDRÁKOVÁ, O. 2011. Two new European species from the heterogeneous Caloplaca holocarpa group (Teloschistaceae). The Lichenologist 44: 73-89.

Recibido el 13 de agosto de 2014, aceptado el 4 de diciembre de 2014. 
